\title{
Modified Exponential Ratio and Product Estimators for Finite Population Mean in Double Sampling
}

\author{
Housila P. Singh and Gajendra K. Vishwakarma \\ Vikram University, Ujjain, India
}

\begin{abstract}
This paper presents exponential ratio and product estimators for estimating finite population mean using auxiliary information in double sampling and analyzes their properties. These estimators are compared for their precision with simple mean per unit, usual double sampling ratio and product estimators. An empirical study is also carried out to judge the merits of the suggested estimators.

Zusammenfassung: Diese Arbeit präsentiert exponentielle Verhältnis- und Produktschätzer zur Schätzung des Mittels einer endlichen Population unter Verwendung zusätzlicher Information bei double sampling und analysiert deren Eigenschaften. Diese Schätzer werden auf ihre Präzision mit dem einfachen Mittel und den gewöhnlichen double sampling Verhältnis- und Produktschätzern verglichen. Eine empirische Studie wurde auch durchgeführt, um die Vorteile der vorgeschlagenen Schätzer zu prüfen.
\end{abstract}

Keywords: Auxiliary Variable, Study Variable, Variance.

\section{Introduction}

It is well known that the use of an auxiliary variable $X$ at the estimation stage improves the precision of an estimate of the population mean of a character $Y$ under study. Out of many ratio, product and regression methods of estimation are good examples in this context. When the correlation between study variable $Y$ and auxiliary variable $X$ is positive high, the classical ratio estimator is considered to be the most practicable. The product estimator of Robson (1957), which is rediscovered by Murthy (1964), is employed quite effectively in the case of high negative correlation between study variable $Y$ and auxiliary variable $X$. Further if the relation between $Y$ and $X$ is a straight line passing through the neighborhood of the origin and the variance of $Y$ about this line is proportional to auxiliary variable $X$, the ratio estimator is as good as regression estimator.

However, in many situations of practical importance the regression line does not pass through the neighborhood of the origin. In these situations, ratio estimator does not perform equally well as that of regression estimator. This led various authors including Singh (1965), Gupta (1970, 1978), Sahai (1979), Srivastava (1967, 1970, 1971, 1980, 1981), Reddy (1973, 1974), Walsh (1970), Bandyopadhyay (1980), Srivenkataramana (1980), and Upadhyaya and Singh (1999) to suggest modified ratio estimators in order to provide better alternatives.

Let in a finite population $U=\left\{U_{1}, \ldots, U_{N}\right\}$ of size $N$ the value of the variables on the $i$ th unit $U_{i}, i=1, \ldots, N$, be $\left(y_{i}, x_{i}\right)$. Let $\bar{Y}=\sum_{i=1}^{N} y_{i} / N$ and $\bar{X}=\sum_{i=1}^{N} x_{i} / N$ be the population means of the study variable $y$ and the auxiliary variable $x$, respectively. 
For estimating the population mean $\bar{Y}$ of $y$ a simple random sample of size $n$ is drawn without replacement from the population $U$. Let $\bar{y}=\sum_{i=1}^{n} y_{i} / n$ and $\bar{x}=\sum_{i=1}^{n} x_{i} / n$ be the unbiased estimators of population means $\bar{Y}$ and $\bar{X}$, respectively. Then the classical ratio estimator is defined by

$$
\bar{y}_{R}=\bar{y} \frac{\bar{X}}{\bar{x}}, \quad \text { if } \quad \bar{x} \neq 0
$$

and the product estimator is given by

$$
\bar{y}_{p}=\bar{y} \frac{\bar{x}}{\bar{X}},
$$

where $\bar{X}$, the population mean of the auxiliary variable $x$ is known. With known population mean $\bar{X}$, Bahl and Tuteja (1991) suggested the exponential ratio-type estimator

$$
\hat{\bar{Y}}_{R e}=\bar{y} \exp \left(\frac{\bar{X}-\bar{x}}{\bar{X}+\bar{x}}\right)
$$

and the exponential product-type estimator

$$
\hat{\bar{Y}}_{P e}=\bar{y} \exp \left(\frac{\bar{x}-\bar{X}}{\bar{x}+\bar{X}}\right)
$$

for the population mean $\bar{Y}$.

If the population mean $\bar{X}$ of the auxiliary variable $x$ is not known before start of the survey, then it may be more efficient to do the sampling in two-phase (or double sampling). It is a powerful and cost effective (economical) procedure and hence has eminent role to play in survey sampling, see Hidiroglou and Sarndal (1998); Hidiroglou (2001). It is usually employed when the number of units required to give the desired precision on different items is widely different. This procedure is also useful when it is proposed to use the information gathered in the first phase as auxiliary information in order to increase the precision of the information to be gathered in the second phase. Thus, in a survey to estimate the production of lime crop based on orchards as sampling units, a comparatively larger sample is taken to determine the acreage under the crop while the yield rate is determined from only a sub-sample of the orchards selected for determining acreage, see Sukhatme (1962). As another example, suppose it is considered desirable to select a sample of agricultural holdings with probability proportionate to area, but information on area is not available. We may then decide to take an initial random sample of holdings and collect information on their areas (say, by asking the holders) and then take a sub-sample of holding with probability proportionate to area and collect information on the characters under study from this sub-sample, see Raj (1968). Double sampling is also used if the value of auxiliary variable $x$ is obtained by performing a non destructive experiment where as to obtain the value of study variable $y$ of a unit a destructive experiment has to be performed, see Unnikrishan and Kunte (1995, p.104). Neyman (1938) was the first to formulate double sampling (or two-phase sampling) in connection with collecting information on the strata sizes in a stratified sampling.

The objective of this paper is to propose double sampling versions of Bahl and Tuteja (1991) estimators and study their properties. Throughout the paper simple random sampling without replacement (SRSWOR) scheme has been considered. An empirical study is carried out to demonstrate the performance of the suggested estimators over others. 


\section{Proposed Ratio and Product Estimators}

When the population mean $\bar{X}$ of the auxiliary variable $x$ is unknown, a first-phase sample of size $n^{\prime}$ is drawn from the population on which only the auxiliary variable $x$ is observed. Then a second phase sample of size $n$ is drawn on which both study variable $y$ and auxiliary variable $x$ are observed. Let $\bar{y}=\sum_{i=1}^{n} y_{i} / n$ and $\bar{x}=\sum_{i=1}^{n} x_{i} / n$ denote the sample means of variables $y$ and $x$, respectively, obtained from the second sample of size $n$ and $\bar{x}^{\prime}=\sum_{i=1}^{n^{\prime}} x_{i} / n^{\prime}$ those obtained from the first sample of size $n^{\prime}$. Then the double sampling version of the ratio $\bar{y}_{R d}$ and product $\bar{y}_{P d}$ estimators of population mean $\bar{Y}$ are given by

$$
\bar{y}_{R d}=\bar{y} \frac{\bar{x}^{\prime}}{\bar{x}} \quad \text { and } \quad \bar{y}_{P d}=\bar{y} \frac{\bar{x}}{\bar{x}^{\prime}} .
$$

It is to be mentioned that the estimator $\bar{y}_{R d}$ is due to Sukhatme (1962).

In double (or two-phase) sampling, we suggest the following modified exponential ratio and product estimators for $\bar{Y}$, respectively, as

$$
\hat{\bar{Y}}_{R e M d}=\bar{y} \exp \left(\frac{\bar{x}^{\prime}-\bar{x}}{\bar{x}^{\prime}+\bar{x}}\right) \quad \text { and } \quad \hat{\bar{Y}}_{P e M d}=\bar{y} \exp \left(\frac{\bar{x}-\bar{x}^{\prime}}{\bar{x}+\bar{x}^{\prime}}\right) .
$$

It is easily observed that $\bar{y}_{R d}, \bar{y}_{P d}, \hat{\bar{Y}}_{R e M d}$, and $\hat{\bar{Y}}_{P e M d}$ are biased estimators, but the bias being of the order $n^{-1}$, can be assumed negligible in large samples. It is assumed that the sample size $n$ is large enough so that the biases of the estimators $\bar{y}_{R d}, \bar{y}_{P d}, \hat{\bar{Y}}_{R e M d}$ and $\hat{\bar{Y}}_{P e M d}$ are negligible and the variances of these estimators are obtained up to the terms of order $n^{-1}$, see Srivastava (1970). The following two cases will be discussed:

Case-I when the second phase sample of size $n$ is a sub-sample of the first-phase sample of size $n^{\prime}$, and

Case-II when the second phase sample of size $n$ is drawn independently of the first-phase sample of size $n^{\prime}$, see Bose (1943).

\section{Case-I}

To obtain the variance of the estimators $\hat{\bar{Y}}_{\text {ReMd }}$ and $\hat{\bar{Y}}_{P e M d}$, we write $\bar{y}=\bar{Y}\left(1+e_{0}\right)$, $\bar{x}=\bar{X}\left(1+e_{1}\right)$, and $\bar{x}^{\prime}=\bar{X}\left(1+e_{1}^{\prime}\right)$ such that

$$
\begin{aligned}
& \mathrm{E}\left(e_{0}\right)=\mathrm{E}\left(e_{1}\right)=\mathrm{E}\left(e_{1}^{\prime}\right)=0 \\
& \mathrm{E}\left(e_{0}^{2}\right)=\lambda C_{y}^{2}, \quad \mathrm{E}\left(e_{1}^{2}\right)=\lambda C_{x}^{2}, \quad \mathrm{E}\left(e_{1}^{\prime 2}\right)=\lambda^{\prime} C_{x}^{2} \\
& \mathrm{E}\left(e_{0} e_{1}\right)=\lambda \rho C_{y} C_{x}, \quad \mathrm{E}\left(e_{1} e_{1}^{\prime}\right)=\lambda^{\prime} C_{x}^{2}, \quad \mathrm{E}\left(e_{0} e_{1}^{\prime}\right)=\lambda^{\prime} \rho C_{y} C_{x},
\end{aligned}
$$

where $\lambda=1 / n-1 / N, \lambda^{\prime}=1 / n^{\prime}-1 / N, C_{y}=S_{y} / \bar{Y}, C_{x}=S_{x} / \bar{X}$, and $\rho=S_{y x} /\left(S_{y} S_{x}\right)$ is the correlation coefficient between $y$ and $x, S_{y}^{2}=\sum_{i=1}^{N}\left(y_{i}-\bar{Y}\right)^{2} /(N-1), S_{x}^{2}=$ $\sum_{i=1}^{N}\left(x_{i}-\bar{X}\right)^{2} /(N-1)$, and $S_{y x}=\sum_{i=1}^{N}\left(y_{i}-\bar{Y}\right)\left(x_{i}-\bar{X}\right) /(N-1)$. 
Now expressing $\hat{\bar{Y}}_{R e M d}$ and $\hat{\bar{Y}}_{P e M d}$ in terms of $e^{\prime}$ s we have

$$
\begin{aligned}
& \hat{\bar{Y}}_{\text {ReMd }}=\bar{Y}\left(1+e_{0}\right) \exp \left[\frac{1}{2}\left(e_{1}^{\prime}-e_{1}\right)\left\{1+\frac{1}{2}\left(e_{1}^{\prime}+e_{1}\right)\right\}^{-1}\right] \\
& \hat{\bar{Y}}_{P e M d}=\bar{Y}\left(1+e_{0}\right) \exp \left[-\frac{1}{2}\left(e_{1}^{\prime}-e_{1}\right)\left\{1+\frac{1}{2}\left(e_{1}^{\prime}+e_{1}\right)\right\}^{-1}\right] .
\end{aligned}
$$

Expanding the right hand sides, multiplying out and neglecting the terms of $e^{\prime}$ s greater than or equal to two, we get

$$
\begin{array}{ll}
\hat{\bar{Y}}_{R e M d} \cong \bar{Y}\left[1+e_{0}+\frac{1}{2}\left(e_{1}^{\prime}-e_{1}\right)\right] & \Rightarrow \quad \hat{\bar{Y}}_{R e M d}-\bar{Y}=\bar{Y}\left[e_{0}+\frac{1}{2}\left(e_{1}^{\prime}-e_{1}\right)\right] \\
\hat{\bar{Y}}_{P e M d} \cong \bar{Y}\left[1+e_{0}-\frac{1}{2}\left(e_{1}^{\prime}-e_{1}\right)\right] & \Rightarrow \quad \hat{\bar{Y}}_{P e M d}-\bar{Y}=\bar{Y}\left[e_{0}-\frac{1}{2}\left(e_{1}^{\prime}-e_{1}\right)\right] .
\end{array}
$$

Squaring both sides, taking expectations and using (1), we get their variances to the first degree of approximation as

$$
\begin{aligned}
\operatorname{var}_{I}\left(\hat{\bar{Y}}_{R e M d}\right) & =\bar{Y}^{2} \mathrm{E}\left[e_{0}+\frac{1}{2}\left(e_{1}^{\prime}-e_{1}\right)\right]^{2} \\
& =\bar{Y}^{2} \mathrm{E}\left[e_{0}^{2}+\frac{1}{4}\left(e_{1}^{2}-2 e_{1}^{\prime} e_{1}+e_{1}^{2}\right)+\left(e_{0} e_{1}^{\prime}-e_{0} e_{1}\right)\right] \\
& =S_{y}^{2}\left[\lambda+(a / 4) \lambda^{*}(a-4 \rho)\right] \\
\operatorname{var}_{I}\left(\hat{\bar{Y}}_{P e M d}\right) & =S_{y}^{2}\left[\lambda+(a / 4) \lambda^{*}(a+4 \rho)\right],
\end{aligned}
$$

where $\operatorname{var}_{I}(\cdot)$ stands for the variance in Case- $I, \lambda^{*}=1 / n-1 / n^{\prime}=\lambda-\lambda^{\prime}$, and $a=C_{x} / C_{y}$.

To the first degree of approximation the variances of $\bar{y}_{R d}$ and $\bar{y}_{P d}$ are

$$
\begin{aligned}
& \operatorname{var}_{I}\left(\bar{y}_{R d}\right)=S_{y}^{2}\left[\lambda+\lambda^{*} a(a-2 \rho)\right] \\
& \operatorname{var}_{I}\left(\bar{y}_{P d}\right)=S_{y}^{2}\left[\lambda+\lambda^{*} a(a+2 \rho)\right]
\end{aligned}
$$

and the variance of the usual unbiased estimator $\bar{y}$ under the SRSWOR scheme is

$$
\operatorname{var}(\bar{y})=\lambda S_{y}^{2}
$$

\subsection{Efficiency Comparison}

From (4) and (8) we have $\operatorname{var}(\bar{y})-\operatorname{var}_{I}\left(\hat{\bar{Y}}_{R e M d}\right)=S_{y}^{2}(a / 4) \lambda^{*}(4 \rho-a)$, which is positive if $4 \rho-a>0$, i.e. if

$$
\rho / a>1 / 4
$$

From (4) and (6) we have $\operatorname{var}_{I}\left(\bar{y}_{R d}\right)-\operatorname{var}_{I}\left(\hat{\bar{Y}}_{R e M d}\right)=S_{y}^{2}(a / 4) \lambda^{*}(3 a-4 \rho)$, which is greater than zero if $3 a-4 \rho>0$, i.e. if

$$
\rho / a<3 / 4
$$

Now combining (9) and (10), we observe that exponential ratio estimator $\hat{\bar{Y}}_{R e M d}$ is more efficient than the usual unbiased estimator $\bar{y}$ and the double sampling ratio estimator $\bar{y}_{R d}$, if $1 / 4<\rho / a<3 / 4$, a condition which is usually met in practice. 
From (2) and (8) we have $\operatorname{var}(\bar{y})-\operatorname{var}_{I}\left(\hat{\bar{Y}}_{P e M d}\right)=-S_{y}^{2}(a / 4) \lambda^{*}(4 \rho+a)$, which is positive if $4 \rho+a<0$, i.e. if

$$
\rho / a<-1 / 4 \text {. }
$$

From (5) and (7) we have $\operatorname{var}_{I}\left(\bar{y}_{P d}\right)-\operatorname{var}_{I}\left(\hat{\bar{Y}}_{P e M d}\right)=S_{y}^{2}(a / 4) \lambda^{*}(3 a+4 \rho)$, which is positive if $3 a+4 \rho>0$, i.e. if

$$
\rho / a>-3 / 4 .
$$

Thus combining (11) and (12), we find that $\hat{\bar{Y}}_{P e M d}$ is more efficient than $\bar{y}$ and $\bar{y}_{P d}$ if $-3 / 4<\rho / a<-1 / 4$.

\section{Case-II}

To obtain the variance of the estimators $\hat{\bar{Y}}_{R e M d}$ and $\hat{\bar{Y}}_{P e M d}$ we write $\bar{y}=\bar{Y}\left(1+e_{0}\right)$, $\bar{x}=\bar{X}\left(1+e_{1}\right)$, and $\bar{x}^{\prime}=\bar{X}\left(1+e_{1}^{\prime}\right)$ such that

$$
\begin{aligned}
& \mathrm{E}\left(e_{0}\right)=\mathrm{E}\left(e_{1}\right)=\mathrm{E}\left(e_{1}^{\prime}\right)=0, \\
& \mathrm{E}\left(e_{0}^{2}\right)=\lambda C_{y}^{2}, \quad \mathrm{E}\left(e_{1}^{2}\right)=\lambda C_{x}^{2}, \quad \mathrm{E}\left(e_{1}^{\prime 2}\right)=\lambda^{\prime} C_{x}^{2}, \\
& \mathrm{E}\left(e_{0} e_{1}\right)=\lambda \rho C_{y} C_{x}, \quad \mathrm{E}\left(e_{1} e_{1}^{\prime}\right)=0=\mathrm{E}\left(e_{0} e_{1}^{\prime}\right) .
\end{aligned}
$$

Squaring both sides of (2) and (3), taking expectations and using (13), we get the variances of $\hat{\bar{Y}}_{R e M d}$ and $\hat{\bar{Y}}_{P e M d}$ to the first degree of approximation respectively as

$$
\begin{aligned}
\operatorname{var}_{I I}\left(\hat{\bar{Y}}_{R e M d}\right) & =S_{y}^{2}\left[\lambda+(a / 4)\left\{\lambda(a-4 \rho)+\lambda^{\prime} a\right\}\right] \\
\operatorname{var}_{I I}\left(\hat{\bar{Y}}_{P e M d}\right) & =S_{y}^{2}\left[\lambda+(a / 4)\left\{\lambda(a+4 \rho)+\lambda^{\prime} a\right\}\right]
\end{aligned}
$$

To the first degree of approximation we now have

$$
\begin{aligned}
& \operatorname{var}_{I I}\left(\bar{y}_{R d}\right)=S_{y}^{2}\left[\lambda\{1+a(a-2 \rho)\}+\lambda^{\prime} a^{2}\right] \\
& \operatorname{var}_{I I}\left(\bar{y}_{P d}\right)=S_{y}^{2}\left[\lambda\{1+a(a+2 \rho)\}+\lambda^{\prime} a^{2}\right] .
\end{aligned}
$$

From (8) and (14) we get $\operatorname{var}(\bar{y})-\operatorname{var}_{I I}\left(\hat{\bar{Y}}_{R e M d}\right)=-S_{y}^{2}(a / 4)\left[\lambda(a-4 \rho)+\lambda^{\prime} a\right]$, which is positive if $\lambda(a-4 \rho)+\lambda^{\prime} a>0$, i.e. if

$$
\rho / a>\left(1+\lambda^{\prime} / \lambda\right) / 4 \text {. }
$$

From (14) and (16) we have $\operatorname{var}_{I I}\left(\bar{y}_{R d}\right)-\operatorname{var}_{I I}\left(\hat{\bar{Y}}_{R e M d}\right)=S_{y}^{2}(a / 4)\left[\lambda(3 a-4 \rho)+3 \lambda^{\prime} a\right]$, which is positive if $\lambda(3 a-4 \rho)+3 \lambda^{\prime} a>0$, i.e. if

$$
\rho / a<3\left(1+\lambda^{\prime} / \lambda\right) / 4
$$

If the population is very large, i.e. $N \rightarrow \infty$, then the above conditions reduce to $\rho / a>\left(1+n / n^{\prime}\right) / 4$ and $\rho / a<3\left(1+n / n^{\prime}\right) / 4$.

Thus, we see from (18) and (19) that $\hat{\bar{Y}}_{R e M d}$ is more efficient than $\bar{y}$ and $\bar{y}_{R d}$ if

$$
\left(1+\lambda^{\prime} / \lambda\right) / 4<\rho / a<3\left(1+\lambda^{\prime} / \lambda\right) / 4 .
$$


For large $N$ this simplifies to $\left(1+n / n^{\prime}\right) / 4<\rho / a<3\left(1+n / n^{\prime}\right) / 4$.

From (8) and (15) we have $\operatorname{var}(\bar{y})-\operatorname{var}_{I I}\left(\hat{\bar{Y}}_{P e M d}\right)=-S_{y}^{2}(a / 4)\left[\lambda(a+4 \rho)+\lambda^{\prime} a\right]$, which is greater than zero if $\lambda(a+4 \rho)+\lambda^{\prime} a<0$, i.e. if

$$
\rho / a<-\left(1+\lambda^{\prime} / \lambda\right) / 4 \text {. }
$$

From (15) and (17) we have $\operatorname{var}_{I I}\left(\bar{y}_{P d}\right)-\operatorname{var}_{I I}\left(\hat{\bar{Y}}_{P e M d}\right)=a S_{y}^{2}\left[3 a\left(\lambda+\lambda^{\prime}\right)+4 \rho \lambda\right] / 4$, which is positive if $3 a\left(\lambda+\lambda^{\prime}\right)+4 \rho \lambda>0$, i.e. if

$$
\rho / a>-3\left(1+\lambda^{\prime} / \lambda\right) / 4
$$

For $N \rightarrow \infty,(21)$ and (22) reduce to $\rho / a<-\left(1+n / n^{\prime}\right) / 4$ and $\rho / a>-3\left(1+n / n^{\prime}\right) / 4$.

Now combining (21) and (22), we find that $\hat{\bar{Y}}_{P e M d}$ is better than $\bar{y}$ and $\bar{y}_{P d}$ if

$$
-3\left(1+\lambda^{\prime} / \lambda\right) / 4<\rho / a<-\left(1+\lambda^{\prime} / \lambda\right) / 4 .
$$

If the population is large, this reduces to $-3\left(1+n / n^{\prime}\right) / 4<\rho / a<-\left(1+n / n^{\prime}\right) / 4$.

Further from (6) and (14) we have $\operatorname{var}_{I}\left(\hat{\bar{Y}}_{R e M d}\right)-\operatorname{var}_{I I}\left(\hat{\bar{Y}}_{R e M d}\right)=-\left(S_{y}^{2} a \lambda^{\prime}\right)(a-$ $2 \rho) / 2$, which is greater than zero if $(a-2 \rho)<0$, i.e. if

$$
\rho / a>1 / 2 .
$$

From (7) and (15) we have $\operatorname{var}_{I}\left(\hat{\bar{Y}}_{P e M d}\right)-\operatorname{var}_{I I}\left(\hat{\bar{Y}}_{P e M d}\right)=-\left(S_{y}^{2} a \lambda^{\prime}\right)(a+2 \rho) / 2$, which is greater than zero if $a+2 \rho<0$, i.e. if

$$
\rho / a<-1 / 2 .
$$

Conditions (23) and (24) are usually met in survey situations.

\section{Empirical Study}

To examine the merits of the suggested estimator we have considered five natural population data sets. The description of the population are given below.

Population I: Murthy (1967, p.228)

$x$ : fixed capital, $y$ : output, $N=80, n=10, n^{\prime}=30$,

$$
\bar{Y}=5182.64, C_{y}=0.3542, C_{x}=0.7507, \rho=0.9413, \rho / a=0.44413 .
$$

Population II: Murthy (1967, p.228)

$x$ : number of workers, $y$ : output, $N=80, n=10, n^{\prime}=30$,

$$
\bar{Y}=5182.64, C_{y}=0.3542, C_{x}=0.9484, \rho=0.9150, \rho / a=0.34173 .
$$

Population III: Das (1988)

$x$ : number of agricultural laborers for 1961, $y$ : number of agricultural laborers for 1971, $N=278, n=30, n^{\prime}=70$,

$$
\bar{Y}=39.0680, C_{y}=1.4451, C_{x}=1.6198, \rho=0.7213, \rho / a=0.64351 .
$$


Table 1: Percent relative efficiencies with respect to $\bar{y}$

\begin{tabular}{|c|c|c|c|c|c|}
\hline Case-I & \multicolumn{5}{|c|}{ Population } \\
\hline Estimator & I & II & III & IV & V \\
\hline $\bar{y}_{R d}$ & 72.36 & 36.65 & 130.02 & $*$ & * \\
\hline $\bar{y}_{P d}$ & * & $*$ & $*$ & 94.48 & 103.35 \\
\hline$\hat{\bar{Y}}_{R e M d}$ & 298.63 & 200.23 & 147.03 & $*$ & * \\
\hline$\hat{\bar{Y}}_{P e M d}$ & * & $*$ & $*$ & 123.61 & 111.48 \\
\hline Case-II & \multicolumn{5}{|c|}{ Population } \\
\hline Estimator & I & II & III & IV & V \\
\hline $\bar{y}_{R d}$ & 38.89 & 20.09 & 91.65 & $*$ & 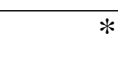 \\
\hline $\bar{y}_{P d}$ & $*$ & * & $*$ & 74.56 & 86.58 \\
\hline$\hat{\bar{Y}}_{R e M d}$ & 252.89 & 130.02 & 161.41 & $*$ & $*$ \\
\hline$\hat{\bar{Y}}_{P e M d}$ & * & $*$ & $*$ & 122.22 & 112.73 \\
\hline
\end{tabular}

Population IV: Steel and Torrie (1960, p.282)

$x$ : chlorine percentage, $y$ : $\log$ of leaf burn in sacs, $N=30, n=4, n^{\prime}=12$,

$$
\bar{Y}=0.6860, C_{y}=0.4803, C_{x}=0.7493, \rho=-0.4996, \rho / a=-0.46680 .
$$

Population V: Dobson (1990, p.47)

$x$ : initial white blood cell count, $y$ : survival time leukemia patient, $N=20, n=4$, $n^{\prime}=8$,

$$
C_{y}=0.2017, C_{x}=0.1502, \rho=-0.4074, \rho / a=-0.54709 .
$$

We have computed the percent relative efficiencies of $\bar{y}, \bar{y}_{R d}, \bar{y}_{P d}, \hat{\bar{Y}}_{R e M d}$, and $\hat{\bar{Y}}_{P e M d}$ with respect to $\bar{y}$ in Case-I and Case-II and the findings are given in Table 1.

\section{Conclusion}

Table 1 clearly indicates that the ratio and product estimator $\hat{\bar{Y}}_{R e M d}$ and $\hat{\bar{Y}}_{P e M d}$ are more efficient than $\bar{y}, \bar{y}_{R d}$, and $\bar{y}_{P d}$. It is also observed that in Case-I the performances of the proposed estimators are better than in Case-II except in population V. Thus, the use of the suggested estimators should be preferred in practice.

\section{Acknowledgements}

The authors are thankful to the editor $\mathrm{H}$. Friedl and the learned referee for their valuable suggestions regarding the improvement of this paper.

\section{References}

Bahl, S., and Tuteja, R. K. (1991). Ratio and product type exponential estimator. Information and Optimization Sciences, 12, 159-163.

Bandyopadhyay, S. (1980). Improved ratio and product estimators. Sankhya, 42, 45-49. 
Bose, C. (1943). Note on the sampling error in the method of double sampling. Sankhya, 6, 330.

Das, A. K. (1988). Contribution to the theory of sampling strategies based on auxiliary information. Unpublished doctoral dissertation.

Dobson, A. J. (1990). An Introduction to Generalized Linear Models. New York: Chapman \& Hall.

Gupta, P. C. (1970). On some estimation problem in sampling using auxiliary information. Unpublished doctoral dissertation.

Gupta, P. C. (1978). On some quadratic and higher degree ratio and product estimators. Jour. Ind. Soc. Agril. Stat., 30, 71-80.

Hidiroglou, M. A. (2001). Double sampling. Survey Methodology, 27, 143-154.

Hidiroglou, M. A., and Sarndal, C. E. (1998). Use of auxiliary information for two-phase sampling. Survey Methodology, 24, 11-20.

Murthy, M. N. (1964). Product method of estimation. Sankhya, A, 26, 69-74.

Murthy, M. N. (1967). Sampling Theory and Methods. Calcutta, India: Statistical Publishing Society.

Neyman, J. (1938). Contribution to the theory of sampling human population. Journal of the American Statistical Association, 33, 101-116.

Raj, D. (1968). Sampling Theory. New York: Mc-Graw Hill.

Reddy, V. N. (1973). On ratio and product method of estimation. Sankhya, B, 35, 307-317.

Reddy, V. N. (1974). On a transformed ratio method of estimation. Sankhya, C, 36, 59-70.

Robson, D. S. (1957). Application of multivariate polykays to the theory of unbiased ratio-type estimation. Journal of the American Statistical Association, 52, 511-522.

Sahai, A. (1979). An efficient variant of the product and ratio estimators. Statistica Neerlandica, 33, 27-35.

Singh, M. P. (1965). On the estimation of ratio and product of population parameters. Sankhya, B, 27, 321-328.

Srivastava, S. K. (1967). An estimator using auxiliary information. Calcutta Statistical Association. Bulletin, 16, 121-132.

Srivastava, S. K. (1970). A two-phase sampling estimator in sample surveys. Australian Journal of Statistics, 12, 23-27.

Srivastava, S. K. (1971). Generalized estimator for mean of a finite population using multiauxiliary information. Journal of the American Statistical Association, 66, 404-407.

Srivastava, S. K. (1980). A class of estimator using auxiliary information in sample surveys. Canadian Journal of Statistics, 8, 253-254.

Srivastava, S. K. (1981). A generalized two-phase sampling estimator. Jour. Ind. Soc. Agril. Stat., 33, 37-46.

Srivenkataramana, T. (1980). A dual to ratio estimator in sample surveys. Biometrika, 67, 199-204.

Steel, R. G. D., and Torrie, J. H. (1960). Principles and Procedures of Statistics. New York: Mc-Graw Hills.

Sukhatme, B. V. (1962). Some ratio-type estimators in two-phase sampling. Journal of the American Statistical Association, 57, 628-632. 
Unnikrishan, N. K., and Kunte, S. (1995). Optimality of analogue of Basu's estimator under double sampling design. Sankhya, B, 57, 103-111.

Upadhyaya, L. N., and Singh, H. P. (1999). Use of transformed auxiliary variable in estimating the finite population mean. Biometrical Journal, 41, 627-636.

Walsh, J. E. (1970). Generalization of ratio estimates for population total. Sankhya, A, 32, 99-106.

\author{
Authors' address: \\ Housila P. Singh and Gajendra K. Vishwakarma \\ School of Studies in Statistics \\ Vikram University \\ Ujjain - 456010, M.P., India \\ E-mails: hpsujn@rediffmail.com and vishwagk@rediffmail.com
}

